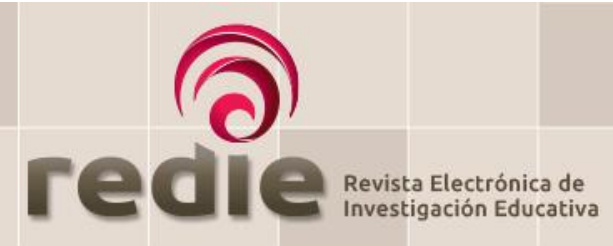

ISSN: $1607-4041$

http://redie.uabc.mx

Vol. 20, Núm. 1, 2018

\title{
Creencias y prácticas de enseñanza de profesores universitarios en Chile
}

\section{Teaching Practices and Beliefs of University Teachers in Chile}

\author{
Elizabeth Gloria Montanares (1) elizabethmontanares@gmail.com \\ Pablo Antonio Junod López (2) pablojunod@gmail.com \\ (1) Universidad Católica de Temuco \\ (2) Universidad Mayor \\ (Recibido: 3 de mayo de 2016; Aceptado para su publicación: 8 de diciembre de 2016)
}

Cómo citar: Montanares, E. G. y Junod, P. A. (2018). Creencias y prácticas de enseñanza de profesores universitarios en Chile. Revista Electrónica de Investigación Educativa, 20(1), 93-103. https://doi.org/10.24320/redie.2018.20.1.1383

\section{Resumen}

Este artículo discute las creencias y prácticas de enseñanza de profesores universitarios en Temuco (Chile). Para ello se aplicó una encuesta con preguntas abiertas a 12 profesores que imparte en los niveles 4o. y 5o. de una carrera del área de la salud. Para el análisis de los datos se utilizó la metodología de la Teoría Fundamentada (TF) y se consideró la codificación abierta, de la cual emergió la categoría Creencias y Prácticas de enseñanza, y cuatro subcategorías: 1) Planificación de la enseñanza, 2) Metodología de enseñanza 3) Modelo epistemológico de enseñanza y 4) Microcurrículum. Los resultados del estudio muestran que dentro de las creencias de los profesores sobre la enseñanza, se valoran prácticas que si bien facilitan que el estudiante participe de forma activa en el aula, son rígidas y están limitadas por elementos como la planificación y los programas previamente construidos; por consiguiente, requieren profundizar en el origen de sus creencias y conocimientos pedagógicos para resignificar su quehacer en el aula y mejorar la educación en este nivel.

Palabras clave: Educación, pedagogía, medios pedagógicos.

\section{Abstract}

This paper discusses the teaching practices and beliefs of university teachers in the city of Temuco in Chile. To that effect, twelve university teachers working with fourth and fifth-level students in a health degree were asked open-ended questions. Data analysis followed the principles of grounded theory (GT) methodology. Open coding was used for the analysis, which produced the category "Teaching practices and beliefs" and four sub-categories: 1) educational planning, 2) teaching methodology, 3) an epistemological model of teaching, and 4) the micro-curriculum. The results of the study show that, as part of their teaching beliefs, teachers place value on practices that make it easier for students to participate and be active in class, but which are also inflexible and restricted by aspects such as planning and previously established programs. Consequently, they need to examine the basis of their beliefs and pedagogical knowledge more thoroughly to inject new meaning into their work in the classroom, thus 
improving education at this level.

Keywords: Education, teaching, teaching methods.

\section{Introducción}

Uno de los temas de discusión en Chile actualmente es sobre el rol de los profesores -tanto en niveles escolares, como en las instituciones de educación superior (IES). Hay acuerdo general sobre la importancia de éstos en los cambios que se implementen con el fin de mejorar la calidad de la educación. El factor "profesor" es una de las variables que mayor peso tienen sobre los resultados de aprendizaje de los estudiantes (Tapia-Gutiérrez, Becerra-Peña, Mansilla-Sepúlveda y Saavedra-Muñoz, 2011). La calidad de la enseñanza se relaciona, entre otras variables, con las características personales y profesionales del docente y con el modo en que enfrenta y conduce el proceso pedagógico (Gajardo y De Andraca, 1992). Esto es complejo debido a los continuos cambios socioculturales y la necesidad de que sean un real aporte a la formación de profesionales idóneos en contextos que se transforman permanentemente. De hecho, Martínez (1999, como se cita en Salinas, 1998) plantea la necesidad de resignificar la docencia universitaria en tiempos de cambios; por su parte, Moreno y Azcárate (2003) señalan que por las necesidades de la sociedad actual -que cuenta con personas más competitivas, y cualificadas- las IES y los profesores deben redefinir sus metas.

Por ello es importante revisar el ejercicio docente en el aula, lugar en el que deben manejar formas de enseñanza exitosas enfocadas a contextos específicos (en ocasiones complejos) (Shulman, 2005). Es el caso de la región de la Araucanía, donde la población presenta rasgos acentuados de diversidad social y cultural. Realidad en la que el profesorado debe desarrollar métodos y estrategias de enseñanza que permitan el aprendizaje de todos los estudiantes. Es así como cobra fuerza la necesidad de transformar la docencia mejorando las estrategias didácticas de los profesores, sus sistemas de comunicación y la distribución de los materiales de aprendizaje.

Las estrategias utilizadas resultan de decisiones que se relacionan en gran medida con las ideas y creencias que ellos poseen, relación presentada por primera vez por Clark y Peterson (1986), quienes afirman que las formas de pensar del docente influyen en sus percepciones sobre los estudiantes, en la planificación de la enseñanza y en su conducta en el aula. Esta idea es corroborada en estudios recientes que señalan que la actuación y concepción del proceso de enseñanza y aprendizaje del profesor se comprende al conocer sus creencias, las que sostienen su praxis pedagógica (Díaz, Martínez, Roa y Sanhueza, 2010). Incluso Roberts, Conner, Estepp, Giorgi y Stripling, (2012) muestran otra arista al sostener que a mayor claridad y definición que el profesor tenga sobre sus creencias, mayor es su éxito como instructor. Por otro lado el hecho de que los profesores orienten su conducta a partir de sus propios conocimientos y creencias (Marcelo, 2001), tendría efectos sobre la actitud de los estudiantes, quienes mostrarían mayor compromiso con su propio aprendizaje en la medida que observan que sus profesores están involucrados en el proceso de enseñanza aprendizaje (Díaz et al., 2010).

En definitiva, el conocimiento de las creencias del profesorado es importante en la medida en que permite a los docentes un mayor grado de consciencia, responsabilidad y control en las elecciones de los modelos epistemológicos de enseñanza utilizados y, por ende, optimiza los aprendizajes de sus estudiantes, los que además podrían enfrentar el proceso con un mayor grado de compromiso.

En razón de lo anterior tanto, los objetivos planteados en esta investigación fueron describir las creencias sobre enseñanza y aprendizaje de profesores universitarios en la ciudad de Temuco, evidenciando el vínculo entre las formas de enseñanza con la diversidad social y cultural de la región. Este conocimiento podría aportar a la mejora de los procesos asociados al acto de enseñar y, con ello, a que los estudiantes alcancen aprendizajes significativos que puedan movilizar hacia sus futuros escenarios laborales y sociales.

En el apartado metodológico se presenta el diseño de investigación, desde el enfoque cualitativointerpretativo conocido como Teoría Fundamentada (TF); los resultados se representan organizados en categorías y subcategorías de análisis. 


\subsection{Creencias}

Llinares (1991) enuncia la naturaleza de las creencias considerándolas en el ámbito de la subjetividad del individuo, a través de las cuales interpreta y decide sobre lo que sucede a su alrededor:

Las creencias son conocimientos subjetivos, poco elaborados, generados a nivel particular por cada individuo para explicarse y justificar muchas de las decisiones y actuaciones personales y profesionales vividas, las creencias no se fundamentan sobre la racionalidad, sino más bien sobre los sentimientos, las experiencias y la ausencia de conocimientos específicos del tema con el que se relacionan, lo que las hace ser muy consistentes y duraderas por cada individuo (p. 110).

Sigel, 1985 y Borg, 2001 destacan como componente principal de las creencias una verdad en la que el individuo cree (aunque reconoce que existen otras distintas) y con base en ella guía sus pensamientos y conducta. De esta manera, hay coincidencia en el carácter individual y subjetivo de las creencias, así como en el papel que juegan en la conducta de cada individuo. Desde otra perspectiva, las creencias son constructos dinámicos y emergentes, cambiantes a medida que tenemos experiencias nuevas, están situadas en un contexto particular, son utilizadas como herramientas para enfrentar problemas, asociadas a contextos específicos y vinculadas a identidades particulares (Barcelos y Kajala 2003, como se cita en Tapia, 2013). Si bien en la mayoría de los autores hay elementos en común en la conceptualización de las creencias, se reconoce la dificultad en definirlas pues, señala Latorre y Blanco (2007), se asocian con diversas nociones: valores, actitudes y opiniones. Quintana (2001) incluso reconoce la importancia de elementos intelectuales, culturales, ambientales, emocionales y de voluntad de creer del individuo.

\subsection{Creencias, concepciones y conocimiento}

Da Ponte (1999) no diferencia entre creencias y concepciones, considera a ambas como elementos fundamentales que estructuran el conocimiento $y$, por consiguiente, forman parte del mismo. Por su parte, García, Azcárate y Moreno (2006), en un estudio que sistematiza la relación entre conocimientos, creencias y concepciones, señalan que las creencias son subjetivas y poco elaboradas: las concepciones son organizadores de las creencias, de las imágenes, conceptos, proposiciones, reglas que afectan lo que se percibe, indican. De esta forma, las concepciones son constructos que contienen las creencias y los conocimientos.

Sobre el concepto de conocimiento, Nisbett y Ross (1980) (como se cita en Pajares, 1992), presentan una estrecha vinculación entre ambos conceptos; para ellos el conocimiento es una estructura compuesta por elementos cognitivos, esquemáticos y organizados, al contrario de las creencias que están compuestas principalmente de evaluaciones y juicios. De esta manera, la comprensión del texto es una instancia de conocimiento cognitivo para el profesor. Pero aquel conocimiento que involucra las capacidades de los estudiantes, o su comportamiento, es parte de otro tipo de conocimiento, catalogable como creencias. Éstas, aun cuando por su subjetividad son menos confiables, pueden afectar al conocimiento cognitivo.

\subsection{Creencias, conocimiento y enseñanza}

Los estudios sobre el pensamiento docente conforman una rama de la investigación educativa que tiene tres objetivos fundamentales: 1) Describir la vida mental de los maestros, 2) comprender y explicar las conductas observables de los docentes antes, durante y después de la práctica educativa y 3) construir un marco general de la psicología cognitiva de la enseñanza que pueda ser usada por investigadores, diseñadores de políticas públicas y currículos, formadores de docentes y los docentes mismos (Clark y Peterson, 1986). Estos autores desarrollan un Modelo de Pensamiento Docente (figura 1) compuesto por elementos como la planificación de la enseñanza, los pensamientos y decisiones involucradas en la acción misma de enseñanza, las reflexiones luego de la enseñanza, el conocimiento y las creencias. 


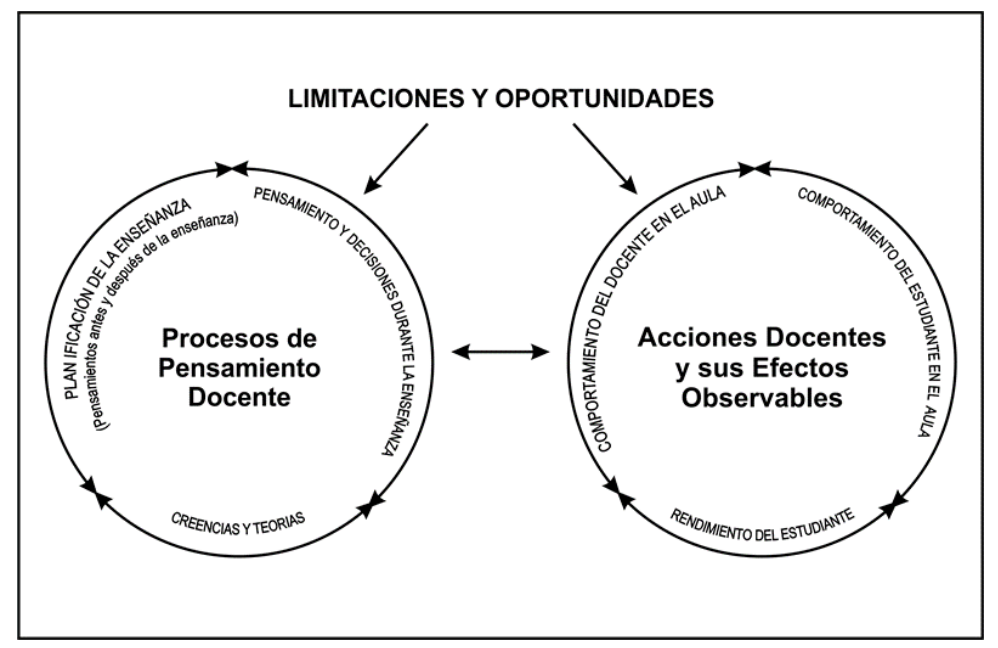

Figura 1. Modelo de pensamiento y acción docente de Clark y Peterson (1986).

Este modelo muestra las relaciones entre los temas centrales para el análisis del pensamiento docente, como son la planificación de la enseñanza, los pensamientos y decisiones involucrados en la acción misma de enseñanza, las reflexiones luego de la enseñanza, el conocimiento y las creencias. Además, visibiliza aspectos relativos a procesos cognitivos y emocionales no observables, en relación con las conductas observables del maestro, que afectan directamente a los estudiantes, como son el comportamiento del profesor en el aula, el rendimiento de los estudiantes y el comportamiento de los mismos.

El avance en estudios sobre creencias y pensamientos del profesorado transforma la concepción del profesor, que hasta entonces respondía a los enfoques conductistas de la enseñanza, a un constructivista, que elabora y que continuamente construye, comprobando su teoría personal del mundo. En este sentido, la propuesta de Chevallard (1997) plantea el concepto de Transposición Didáctica como el proceso a través del cual se transforma un objeto de saber a enseñar en un objeto de enseñanza, donde el profesor juega un papel activo como deformador del conocimiento, con el fin de adaptarlo a la enseñanza.

También Shulman (1993) desarrolla un Modelo de Conocimiento Didáctico del Contenido (CDC), que relaciona los conocimientos relativos a la materia y los conocimientos didácticos. Lo que implica una gran cantidad de conocimientos que el profesor debe poseer. En estudio previo, Gudmundsdottir (1991) resaltó como un componente del CDC, las concepciones, valores, y creencias de lo que significa enseñar una materia en un contexto determinado. En definitiva, las creencias y el conocimiento del contenido que los profesores poseen están vinculados, y son tan poderosos e influyentes, que es necesario que los profesores reconozcan las influencias que aquellas creencias tienen sobre lo que aprenden y lo que enseñan (Shulman, 2005).

\subsection{Estrategias de enseñanza}

Las estrategias didácticas, conjunto de procedimientos apoyados en técnicas de enseñanza que tienen por objeto llevar a buen término la acción didáctica y alcanzar los objetivos de aprendizaje, están relacionadas con las creencias y conocimientos del profesor. Según Shulman (1993), forman parte del conocimiento del contenido pedagógico, el que permite que la asignatura a enseñar sea comprensible para los demás (Putnam y Borko, 2000). Por su parte, García-Cabrero, Loredo y Carranza (2008) sostienen que este contenido comprende además la concepción global de la docencia de una asignatura, el conocimiento de las interpretaciones, el pensamiento y el aprendizaje de los estudiantes de una asignatura, y el conocimiento del currículo.

Las estrategias de enseñanza son dependientes de los paradigmas de enseñanza conscientes o inconscientes a los que el profesor se adscribe. En esta línea, en un estudio sobre profesores de ciencias, Contreras (2010) resalta las teorías implícitas que los profesores poseen sobre el aprendizaje, 
concluyendo que predominan tendencias más tradicionales, con un concepto de aprendizaje centrado en el contenido, en las explicaciones y en una relación simple entre conocimiento previo y el nuevo conocimiento. Rodrigo et al. (1993, como se cita en De Vicenzi, 2009, p. 90) definen las teorías implícitas sobre la enseñanza como teorías pedagógicas personales reconstruidas sobre la base de conocimientos pedagógicos históricamente elaborados y transmitidos a través de la formación y en la práctica pedagógica. Por lo tanto, son una síntesis de conocimientos culturales y experiencias personales.

Fernández (2012) propone un modelo para la tipificación de las concepciones sobre la enseñanza aprendizaje de profesores del área de la salud. Considera tres estilos elaborados a partir de los planos epistemológicos sobre la relación sujeto/objeto: plano profesor-alumno, profesor-objeto y alumnoobjeto. El plano epistemológico profesor-alumno se caracteriza por la clase expositiva, en donde el docente tiene el control en la conducción de los aprendizajes del alumno; sin embargo, y en ausencia del objeto, no es posible lograr aprendizajes significativos. El plano profesor-objeto considera la interacción del profesor con el objeto de estudio, mientras el alumno observa, apropiándose de los aprendizajes por la memorización; este tipo de docente tampoco logra generar aprendizajes completos, ya que el estudiante no forma parte de la construcción de su propio conocimiento. En el plano epistemológico alumno-objeto, a diferencia de los anteriores, sí se contempla la participación del estudiante con su objeto de estudio, mientras el profesor es un guía o mediador que conduce el proceso del alumno, de esta forma se logra la transformación de la relación sujeto/objeto facilitando el proceso de aprendizaje en base a la experiencia propia.

Por último, Hashweh (1996) relaciona la formación del profesor con sus prácticas, diferenciando a profesores con formación científica, quienen sostendrían creencias más constructivistas o sofisticadas, detectando las concepciones alternativas de los alumnos y empleando estrategias más efectivas, pues poseen un repertorio mayor de estrategias de enseñanza.

\section{Método}

En la investigación se empleó una metodología cualitativo-interpretativa con el enfoque metodológico de la TF (Glaser y Strauss, 1967; Strauss y Corbin, 2002). Según Campo y Labarca (2009), esta teoría fue desarrollada por Glaser y Strauss en 1967 y representa una de las principales tradiciones de la investigación cualitativa en sociología y el interaccionismo simbólico. El método de estudio correspondió a un estudio de caso, pues se trata de investigación empírica que estudia un fenómeno dentro de su contexto de la vida real; se basa en diferentes fuentes de evidencia y sus resultados favorecen el desarrollo de proposiciones teóricas.

Este enfoque permite crear propuestas teóricas basándose exclusivamente en los datos. Es así como en esta investigación se ha puesto especial énfasis en los discursos de los actores plasmados en los textos, ya que son estos los que conforman el material empírico real y, por ende, constituyen la base para desarrollar la teoría.

Con esta apuesta metodológica, y a partir de los datos obtenidos se tuvo acceso a los procesos subjetivos de los docentes, permitiendo la construcción de conocimientos teóricos sobre las creencias asociadas a los procesos de enseñanza en el contexto universitario de la región de la Araucanía.

El tamaño de la muestra está relacionado con la metodología ya mencionada; es decir, corresponde a una de las partes del proceso de recolección de los datos y su análisis. Por ello, la muestra inicial se conformó a partir de 12 casos de profesores de una universidad en la región de la Araucanía, que ejercen docencia en 4o. y 5o. nivel de una carrera del área de la salud. Cumplieron con los siguientes criterios de inclusión para el estudio: a) formación en docencia universitaria b) haber realizado docencia universitaria un mínimo de 5 años. La recolección de la muestra se realizó a través de una entrevista semiestructurada a seis profesores, una vez aplicada esta técnica se realizó un primer análisis de las categorías. Luego de este análisis preliminar, y siguiendo la metodología de la TF, se realizó un muestreo teórico (seleccionando nuevos casos a estudiar según su potencial) para refinar o expandir los conceptos y teorías ya desarrollados. En esta segunda etapa se realizaron otras seis entrevistas. 
El análisis de los datos se realizó siguiendo los pasos propuestos por la TF, lo que permitió construir modelos conceptuales con respecto al fenómeno estudiado. La primera etapa, de codificación abierta, consistió en el examen y fragmentación de la información recopilada para organizar los materiales en conceptos y categorías, proceso en el que el investigador aborda el texto para develar conceptos, ideas y sentidos. Sobre esto, Strauss y Corbin (2002) señalan que para descubrir y desarrollar los conceptos se debe abrir el texto y exponer los pensamientos, ideas y significados contenidos en él. Durante este proceso los datos fueron descompuestos, examinados y comparados en términos de sus similitudes y diferencias, al amparo de una aproximación inductiva a la realidad, por lo que no precisa de una teoría para aplicar conceptos, leyes o dimensiones en el texto que se está codificando; el investigador debe zambullirse en el texto para identificar los conceptos, dimensiones y construir categorías y subcategorías pertinentes a la investigación.

\section{Discusión}

Desde la codificación abierta emergió la categoría Creencias y Prácticas de Enseñanza. Esta red conceptual presenta los siguientes códigos: 1) dominio del contenido, 2) estrategias de motivación, 3) énfasis microcurricular, 4) identidad epistemológica curricular, 5) estrategias didácticas y 6) programación de clases.

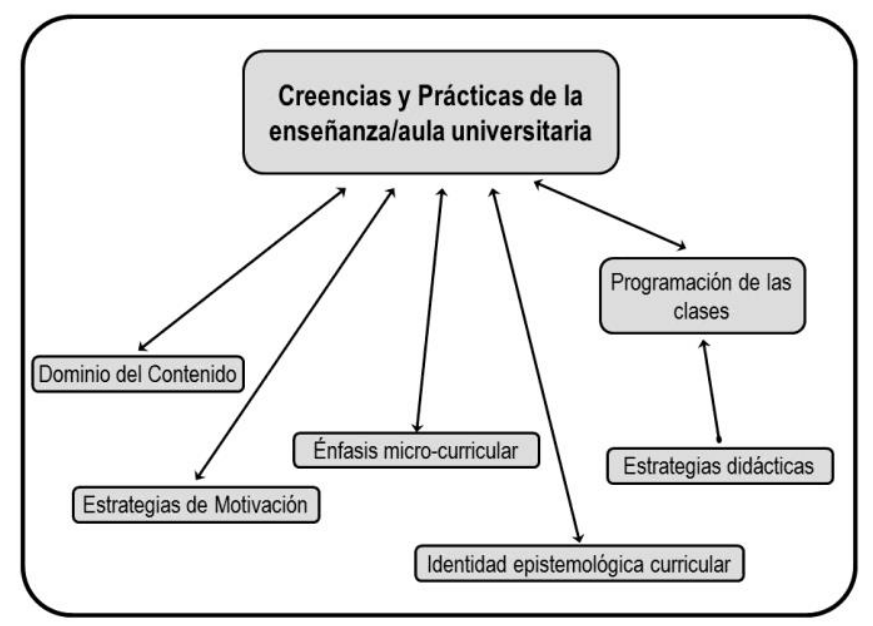

Figura 2. Creencias y prácticas de enseñanza/aula universitaria

Consiste en los constructos que los docentes poseen sobre aspectos asociados a la enseñanza en el contexto del aula universitaria. Es importante tener en cuenta que estas construcciones contienen saberes teóricos, experiencias prácticas y aspectos asociados a la emocionalidad, provocando en ocasiones contradicciones entre las subcategorías.

Dominio del contenido. En esta subcategoría los participantes explicitan que es necesario que el profesor enfatice en el tratamiento de los contenidos para lograr que los estudiantes aprendan. Independientemente de las estrategias utilizadas por el profesor en el logro de los aprendizajes, aspecto que no es identificado por los entrevistados dentro de esta categoría:

Las técnicas de enseñanza son importantes para el aprendizaje de nuestros alumnos, pero no por ello vamos a perder el norte que, a mi parecer, son los conocimientos que nosotros queremos entregar o que queremos que el alumno integre. (E1:010).

Esta lógica es reafirmada con lo señalado por otro entrevistado:

(...) doy prioridad al contenido a enseñar para no dejar temas sin tratar. (E5:010). 
En este sentido, esta disociación entre dominio del contenido y estrategias de enseñanza es uno de los principios que sustentan las creencias del profesorado universitario con respecto a la enseñanza, y que conduciría a un modelo de enseñanza descontextualizada. Al respecto, Shulman (1993) plantea el concepto de conocimiento didáctico del contenido (CDC) como un tipo de conocimiento específico que desarrolla el profesor para enseñar la materia, en el que se combinan tanto los conocimientos de la materia y el conocimiento pedagógico y didáctico relativo a cómo enseñarla (Marcelo, 2001).

Estrategias de motivación. Para los docentes la motivación es central en el proceso de enseñanza aprendizaje; sin embargo, no hay alusión a estrategias específicas de motivación para lograr éxito en los aprendizajes de los estudiantes. La motivación se define más bien como una actitud positiva de los estudiantes sin reconocer que también podría ser resultado de una metodología y estrategias de enseñanza mediadas por el profesor. Esta evidencia se visualiza en las siguientes intervenciones:

Le doy extrema importancia, un estudiante motivado es motor fundamental en el logro de competencias generales y específicas de la carrera. (E7:020).

Un rol fundamental para que se produzca el aprendizaje es tanto la motivación del estudiante como su proactividad, esfuerzo y fundamento para tomar decisiones. (E8:020).

La visión de la motivación es similar a la del constructo anterior en cuanto a que, al igual que el conocimiento, la motivación se entiende como independiente del actuar del profesor y sus decisiones pedagógicas en el proceso enseñanza aprendizaje. Al respecto, Alonso-Tapia (2005) señala:

(...) a veces no es que los alumnos no aprendan porque no estén motivados, sino que no están motivados porque no aprenden, y no aprenden porque su modo de pensar al afrontar las tareas es inadecuado, impidiendo la experiencia satisfactoria que supone sentir que se progresa, experiencia que activa la motivación. (p. 4).

Se hace necesario trabajar sobre el concepto de motivación, estrategias concretas y reflexión sobre las propias prácticas pedagógicas, reconociendo que es de competencia del estudiante, pero principalmente del profesor.

Énfasis microcurricular. En esta subcategoría el foco está puesto en avanzar según lo planificado, esto es, el tratamiento de todos los contenidos y cumplimiento del programa propuesto. Lo anterior es para los profesores la meta final, y requisito infranqueable para aprobar el curso. Esto probablemente no está exento de tensiones, en momentos se deben pasar por alto situaciones complejas asociadas a tiempo, dificultades con la comprensión de contenidos, y otras necesidades del estudiantado. Esto se expresa en las siguientes intervenciones:

Hay que poner atención al avance curricular necesario para la aprobación de la asignatura. (E11:030).

Le otorgo la mayor importancia al contenido del cual se trata la clase. (E6:030).

Lo declarado se opone a lo propuesto por Giroux (1990), quien considera al profesor como:

Un profesional con conciencia social que debería convertirse a sí mismo en un intelectual transformativo, en un agitador social con una concepción del mundo transformadora y con un conocimiento de toda la ciencia, cultura y tecnología moderna en beneficio de la transformación de las sociedades en función a una mejora con un rumbo consciente y planificado.

Por esto es necesario poner atención al rol del profesor como experto (Shulman,1993), y de esa forma al lugar que se le otorga al aprendizaje de los contenidos por parte de los estudiantes versus el cumplimiento de los programas, atendiendo a que este último aspecto también forma parte del éxito del proceso y responde a necesidades curriculares y disciplinares de cada carrera. 
Identidad epistemológica curricular. En este caso podría observarse un vínculo con la subcategoría anterior, pues los textos muestran que se da menor relevancia a la participación de los estudiantes priorizando un actuación mas bien pasiva, con un profesor que vive su rol desde lo tradicional, adoptando el paradigma conductista de enseñanza para lograr los objetivos propuestos:

La mayoría de las ocasiones utilicé metodología de clases expositiva de forma tradicional. Sólo hace algún tiempo y dados los cambios he tendido a modificar las prioridades, sin embargo, intercalo con la cátedra tradicional. (E1:040).

Hay indicios de que existe la creencia entre el profesorado de que las clases tradicionales les permiten jerarquizar los contenidos y darles orden:

Priorizo las clases expositivas, las que utilizo para dar la visión global de un tema, para ordenar contenidos y así ellos, con su propio trabajo, van profundizando. (E7:040).

Existen ideas distintas, pero manteniendo aquella en donde el profesor es el que entrega conocimiento:

(...) priorizo el trabajo autónomo, pero utilizo las clases expositivas para entregar contenidos de base. (E3:040).

Lo declarado por los sujetos revela la inexistencia de un componente racional en la elección del paradigma de enseñanza, lo que hace reflexionar sobre la necesidad de desdibujar la noción de continuidad entre el conocimiento común y el conocimiento científico. Son creencias que impredirían al estudiante trascender del conocimiento abstracto a lo concreto, de la experiencia sensible a lo inteligible (Bachelard, 1973).

Estrategias didácticas. Con respecto a esta subcategoría se observa un contraste con los constructos anteriores, ya que los entrevistados reconocen promover prácticas que indican protagonismo de los estudiantes, evidenciando un reconocimiento de la importancia de distintas estrategias en los procesos de aprendizaje en el aula universitaria con una alta participación de los jóvenes. Este aspecto se reitera en declaraciones como las siguientes:

Participación oral en clases, talleres de 4 a 5 estudiantes donde ellos exponen al azar y preparan un informe escrito. (E10:050).

Preguntas y respuestas, se les envían papers del tema tratado. (E1:050).

Está claro que si bien los docentes dan gran importancia al cumplimiento de los programas y para ello priorizan las clases expositivas, también reconocen el valor de la participación de los estudiantes a través de la ejecución de instancias como las exposiciones orales y los informes escritos, lo que también se incluye en la praxis, siempre a través de su mediación.

Programación de clases. En esta subcategoría los participantes explicitan que el elemento central en la programación de clases es la planificación anticipada, la que estaría alojada en los programas de cada asignatura. Hay una valoración de estos programas, lo que los valida:

Me baso en la Planificación de la Asignatura. (E3:060).

Programa, porque se ha discutido previamente este programa, aun cuando las experiencias anteriores pueden ser utilizadas. (E8:060).

Los contenidos y organización se encuentran en las primeras planificaciones, en parte a veces considero experiencias anteriores. (E11:060).

En este sentido los estudiantes, sus características o nuevos contextos no son reafirmados como parte central de la programación de las clases, lo que ya en 1997 era discutido por Del Carmen, al afirmar que 
es frecuente que al iniciar la enseñanza de nuevos contenidos se parta de cero, ignorando los aprendizajes realizados anteriormente por los alumnos, tanto dentro como fuera de la escuela. Esto tiene varias consecuencias negativas: ignorancia del nivel de partida, lo que puede provocar desajustes importantes; dificultad para establecer relaciones entre lo que los alumnos ya saben y los nuevos contenidos; desaprovechamiento de las experiencias y motivaciones previas (Del Carmen, 1997).

Lo declarado por los sujetos demuestra que las creencias y concepciones que tienen sobre el aprendizaje son inconscientes y se traducen en contradicciones, las que se ven en los relatos sobre la importancia que le dan a procesos de motivación y la forma en que organizan la participación de los estudiantes, que afectan la forma de enseñar y de planificar. La forma de enseñar está basada en la suposición de que el profesor es el que conoce y el estudiante recibe este conocimiento.

\section{Conclusiones}

A partir de este estudio es posible constatar que las creencias de docentes universitarios en la región de la Araucanía con respecto a la enseñanza se presentan como juicios (Nisbett y Ross, 1980) cuando evalúan su propio rol como fundamental al momento de la adquisición de contenidos y conocimientos por parte de los estudiantes. Esto podría ser una situación muy favorable si no se observara rigidez al indicar que el cumplimiento de los programas es garantía fundamental para el éxito del proceso de enseñanza. Se niegan aspectos centrales como el contexto sociocultural en el que se enseña, las características de los estudiantes, y el uso masivo de las tecnologías de la información en los procesos de enseñanza.

No se observa el concepto de resignificación de la docencia, adecuándola a la realidad y características de quienes aprenden (Brunner,1998; Bravo, 2003; Shulman, 1993); sin embargo, emergen aspectos de conocimiento pedagógico (Shulman,1993) al reconocer estrategias de enseñanza, que instalan a los estudiantes en un rol activo. Esto implica, entre otros aspectos, la necesidad de profundizar en investigaciones contextualizadas que visibilicen las creencias del profesorado y su efecto en las propias prácticas pedagógicas con el fin de que puedan construir metodologías y estrategias adecuadas a la realidad y entorno de los estudiantes que, en el futuro, podrían movilizar los aprendizajes hacia sus futuros escenarios laborales y sociales para transformarlos.

\section{Referencias}

Alonso-Tapia, J. (2005). Motivación para el aprendizaje: la perspectiva de los alumnos. En M. Pérez Solís, (Ed), La orientación escolar en centros educativos (pp. 209.242). Madrid: Ministerio de Educación y Ciencia.

Borg, M. (2001). Teacher's beliefs. Key concepts in English Language Teaching (ELT). ELT Journal, 55(2), 186187.

Bachelard, G. (1973). Epistemología. Barcelona: Anagrama.

Campo-Redondo, M. y Labarca, C. (2009). La teoría fundamentada en el estudio empírico de las representaciones sociales: un caso sobre el rol orientador del docente. Revista Opción, 25(60), 41-54.

Chevallard, Y. (1997). La transposición didáctica. Del saber sabio al saber enseñado. Buenos Aires: Aique.

Clark, C. P. y Peterson, B. P. (1986). Teacher's thought processes. Handbook of research on teaching. Nueva York: Macmillan.

Contreras, S. (2010). Las creencias curriculares de los profesores de ciencias: una aproximación a las teorías implícitas sobre el aprendizaje. Horizontes Educacionales, 15(1), 23-36.

Da Ponte, J. (1999). Las creencias y concepciones de maestros como un tema fundamental en formación de maestros. En K. Krainer y F. Goffree (Eds.), On research in teacher education: from a study of teaching 
practices to issues in teacher education (pp. 43-50). Osnabrück: Forschungsintitut für Mathematikdidaktik.

De Vicenzi, A. (2009). Concepciones de la enseñanza y su relación con las prácticas docentes: un estudio con profesores universitarios. Educación y educadores, 12(2) 87-101.

Del Carmen, L. (Coord.) (1997). La enseñanza y el aprendizaje de las ciencias de la naturaleza en la educación secundaria (Vol. 9). Barcelona: Universidad de Barcelona/ Horsori.

Díaz, C., Martínez, P., Roa, I. y Sanhueza, M. G. (2010). Los docentes en la sociedad actual: sus creencias y cogniciones pedagógicas respecto al proceso didáctico. Polis Revista Latinoamericana, 9(25), 421-436.

Fernández, J. A. (2012). La triangulación epistemológica en la interpretación del proceso de enseñanzaaprendizaje de la medicina. Educación Médica Superior, 26(3), 459-466.

Gajardo, M. y de Andraca, A. M. (1992). Docentes y docencia: las zonas rurales. Chile: UNESCO/FLACSO.

García, L., Azcárate, C. y Moreno, M. (2006). Creencias, concepciones y conocimiento profesional de profesores que enseñan cálculo diferencial a estudiantes de ciencias económicas. Revista Latinoamericana de Investigación en Matemática Educativa, 9(1), 85-116.

García-Cabrero, B., Loredo, J. y Carranza, G. (2008). Análisis de la práctica educativa de los docentes: pensamiento, interacción y reflexión. Revista Electrónica de Investigación Educativa, 10(Especial), 1-15. Recuperado de https://redie.uabc.mx/redie/article/view/200

Giroux, H. (1990). Los profesores como intelectuales: hacia una pedagogía crítica del aprendizaje. Barcelona: Paidós.

Giroux, H. (1986). Más allá de la teoría de la correspondencia. En "La nueva sociología de la Educación", Antología. México: Ediciones El caballito.

Glaser, B. G. y Strauss, A. L. (1967). The discovery of grounded theory: strategies for qualitative research. Chicago: Transaction Publishers.

Gudmundsdottir, S. (1991). Story-maker, story-teller: narrative structures in curriculum. Journal of Curriculum Studies, 23(3), 207-218.

Hashweh, M. Z. (1996). Effects of science teachers' epistemological beliefs in teaching. Journal of Research in Science Teaching, 33(1), 47-63.

Latorre, M. y Blanco, F. (2007). Algunos conceptos clave en torno a las creencias de los docentes en formación. Docencia e Investigación, 32(17), 147-170.

Llinares, S. (1991). La formación de profesores de matemáticas. Sevilla: GID- Universidad de Sevilla.

Marcelo, C. (2001). El aprendizaje de los formadores en tiempos de cambio. La aportación de las redes y el caso de la red andaluza de profesionales de la formación. Profesorado: Revista de Currículum y Formación del Profesorado, 5(1), 29-44.

Moreno, M. y Azcárate, C. (2003). Concepciones y creencias de los profesores universitarios de matemáticas acerca de la enseñanza de las ecuaciones diferenciales. Enseñanza de las Ciencias: Revista de Investigación y Experiencias Didácticas, 21(2), 265-280.

Pajares, M. F. (1992). Teachers' beliefs and educational research: cleaning up a messy construct. Review of Educational Research, 62(3), 307-332. 
Putnam, R. y Borko, H. (2000). What do new views of knowledge and thinking have to say about research on teacher learning? Educational Researcher, 29(1), 4-15.

Quintana, J. M. (2001). Las creencias y la educación. Barcelona: Herder.

Roberts, T., Conner, N., Estepp, C., Giorgi, A. y Stripling, C. (2012). Examining the teaching behaviors of successful teachers in a college of agricultural and life sciences. North American Colleges and Teachers of Agriculture Journal, 56(2), 21-28. Recuperado de http://www.jstor.org/stable/nactajournal.56.2.21

Salinas, J. (1998). El rol del profesorado universitario ante los cambios de la era digital. Agenda Académica, 5(1), 143-158.

Shulman, L. (1993). Forum: Teaching as community property; putting an end to pedagogical solitude. Change: The Magazine of Higher Learning, 25(6), 6-7.

Shulman, L. (2005). Signature pedagogies in the professions. Daedalus, 134(3), 52-59.

Sigel, I. (1985). A conceptual analysis of beliefs. En I. E. Sigel (Ed.), Parental belief systems. The psychological consequences for children (pp.345-371). Hillsdale, NJ: Erlbaum.

Strauss, A. y Corbin. J. (2002). Bases de la investigación cualitativa. Técnicas y procedimientos para desarrollar la teoría fundamentada. Bogotá: Contus.

Tapia, R. (2013). Pre-service teachers beliefs about the roles of thesis supervisors: a case study. Gist: Revista Colombiana de Educación Bilingüe, 7, 74-92.

Tapia-Gutiérrez, C., Becerra-Peña, S., Mansilla-Sepúlveda, J. y Saavedra-Muñoz, J. (2011). Liderazgo de los directivos docentes en contextos vulnerables. Educación y educadores, 14(2), 389-409.

Nisbett, R. y Ross, L. (1980). Human inference. Strategies and shortcomings of social judgement. Englewood Cliffs, NJ: Prentice Hall. 